\title{
Depletion of resting zone chondrocytes during growth plate senescence
}

\author{
Lenneke Schrier, Sandra P Ferns, Kevin M Barnes, \\ Joyce A M Emons, Eric I Newman, Ola Nilsson \\ and Jeffrey Baron* \\ Developmental Endocrinology Branch, National Institute of Child Health and Human Development, National Institutes of Health, CRC, Room 1-3330, \\ 10 Center Drive, MSC 1103, Bethesda, Maryland 20892, USA \\ (Requests for offprints should be addressed to J Baron; Email: Jeffrey.baron@nih.gov) \\ *(J Brown is a Commissioned Officer in the United States Public Health Service)
}

\begin{abstract}
With age, the growth plate undergoes senescent changes that cause linear bone growth to slow and finally cease. Based on previous indirect evidence, we hypothesized that this senescent decline occurs because growth plate stem-like cells, located in the resting zone, have a finite proliferative capacity that is gradually depleted. Consistent with this hypothesis, we found that the proliferation rate in rabbit resting zone chondrocytes (assessed by continuous 5-bromo-2'-deoxy-uridine labeling) decreases with age, as does the number of resting zone chondrocytes per area of growth plate.

Glucocorticoid excess slows growth plate senescence. To explain this effect, we hypothesized that glucocorticoid inhibits resting zone chondrocyte proliferation, thus conserving their proliferative capacity. Consistent with this hypothesis, we found that dexamethasone treatment decreased the proliferation rate of rabbit resting zone
\end{abstract}

chondrocytes and slowed the numerical depletion of these cells. Estrogen is known to accelerate growth plate senescence. However, we found that estradiol cypionate treatment slowed resting zone chondrocyte proliferation.

Our findings support the hypotheses that growth plate senescence is caused by qualitative and quantitative depletion of stem-like cells in the resting zone and that growth-inhibiting conditions, such as glucocorticoid excess, slow senescence by slowing resting zone chondrocyte proliferation and slowing the numerical depletion of these cells, thereby conserving the proliferative capacity of the growth plate. We speculate that estrogen might accelerate senescence by a proliferation-independent mechanism, or by increasing the loss of proliferative capacity per cell cycle.

Journal of Endocrinology (2006) 189, 27-36

\section{Introduction}

In humans, the rate of longitudinal bone growth, and hence the rate of overall linear growth, decreases with age. The prenatal linear growth rate exceeds $100 \mathrm{~cm} /$ year. However, by the time of birth, the growth rate has fallen to about $50 \mathrm{~cm} /$ year, and at the age of 10 years the growth rate is about $5 \mathrm{~cm} /$ year. This decline is briefly interrupted by a pubertal growth spurt, after which the deceleration resumes and the growth rate approaches zero (Tanner \& Davies 1985). In other mammals, a similar dramatic decline in growth rate occurs, but without a superimposed pubertal growth spurt.

Kember and Walker (1971) showed that this senescent decline in growth rate is due, in large part, to a decrease in the proliferation rate of growth plate chondrocytes. These functional senescent changes are accompanied by structural senescent changes. There is a gradual decline in the overall growth plate height, proliferative zone height, hypertrophic zone height, size of hypertrophic chondrocytes, and column density (Kember \& Walker 1971, Kember 1973, Masoud et al. 1986a, Masoud et al. 1986b, Walker \& Kember 1972a, Walker \& Kember 1972b, Hunziker \& Schenk 1989, Farquharson \& Loveridge 1990, Weise et al. 2001). The growth plate undergoes ossification as the final step in the senescence program (Weise et al. 2001).

Growth plate senescence appears to be caused by a mechanism intrinsic to the growth plate (Nilsson \& Baron 2004). Previous studies suggest that information about the prior growth history is retained within the growth plate and influences subsequent growth plate structure and function (Gafni et al. 2001). The cell most likely to carry this information is the resting zone chondrocyte because it is the only cell involved in the growth process that remains in the growth plate over long periods of time. 
Any information carried by the proliferative/hypertrophic chondrocytes would presumably be lost when these cells reach the metaphyseal border of the growth plate, undergo apoptosis, and are replaced by bone (Farnum \& Wilsman 1987).

We have recently shown evidence that the resting zone chondrocytes serve as a pool of stem-like cells that generate columnar clones of proliferative zone chondrocytes (Abad et al. 2002). We have also found evidence suggesting that growth plate senescence is not a function of time per se but rather of the cumulative number of divisions that the chondrocytes have undergone (Gafni et al. 2001). Taken together, these previous findings suggest the following model: 1) stem-like cells in the resting zone have a finite proliferative capacity which is gradually exhausted; 2) as this proliferative capacity is exhausted, the proliferation rate of the proliferative zone chondrocytes (which are derived from the stem-like cells) decreases, causing longitudinal bone growth to slow with age and eventually cease.

This depletion of the stem-like cells in the resting zone could be qualitative and/or quantitative; with age there could be a decrease in the proliferative capacity per stem-like cell and/or a decrease in the number of stemlike cells. However, little is known about these chondrocytes. Even their proliferation rate has not been previously measured, perhaps because it is too slow to be assessed by pulse labeling approaches.

Based on our model, we hypothesized that the proliferation rate of resting zone chondrocytes decreases with age, reflecting their diminishing proliferative capacity. We have previously shown evidence that glucocorticoid slows growth plate senescence (Gafni et al. 2001). Therefore, we further hypothesized that glucocorticoid acts by slowing resting zone chondrocyte proliferation, thus conserving their proliferative capacity and hence slowing senescence. In contrast, estrogen accelerates growth plate senescence (Weise et al. 2001), raising the possibility that estrogen might stimulate resting zone chondrocyte proliferation and thus accelerate depletion of their proliferative capacity.

\section{Materials and Methods}

\section{Animal experiments}

New Zealand White (NZW) male rabbits (Covance Research Products, Denver, PA, USA) were housed and cared for in accordance with the Guide for the Care and Use of Laboratory Animals (National Research Council 2003). The experimental procedures were approved by the Animal Care and Use Committee (National Institute of Child Health and Human Development, National Institutes of Health, USA). All animals received National Institutes of Health open formula rabbit ration (NIH 32) and water ad libitum.

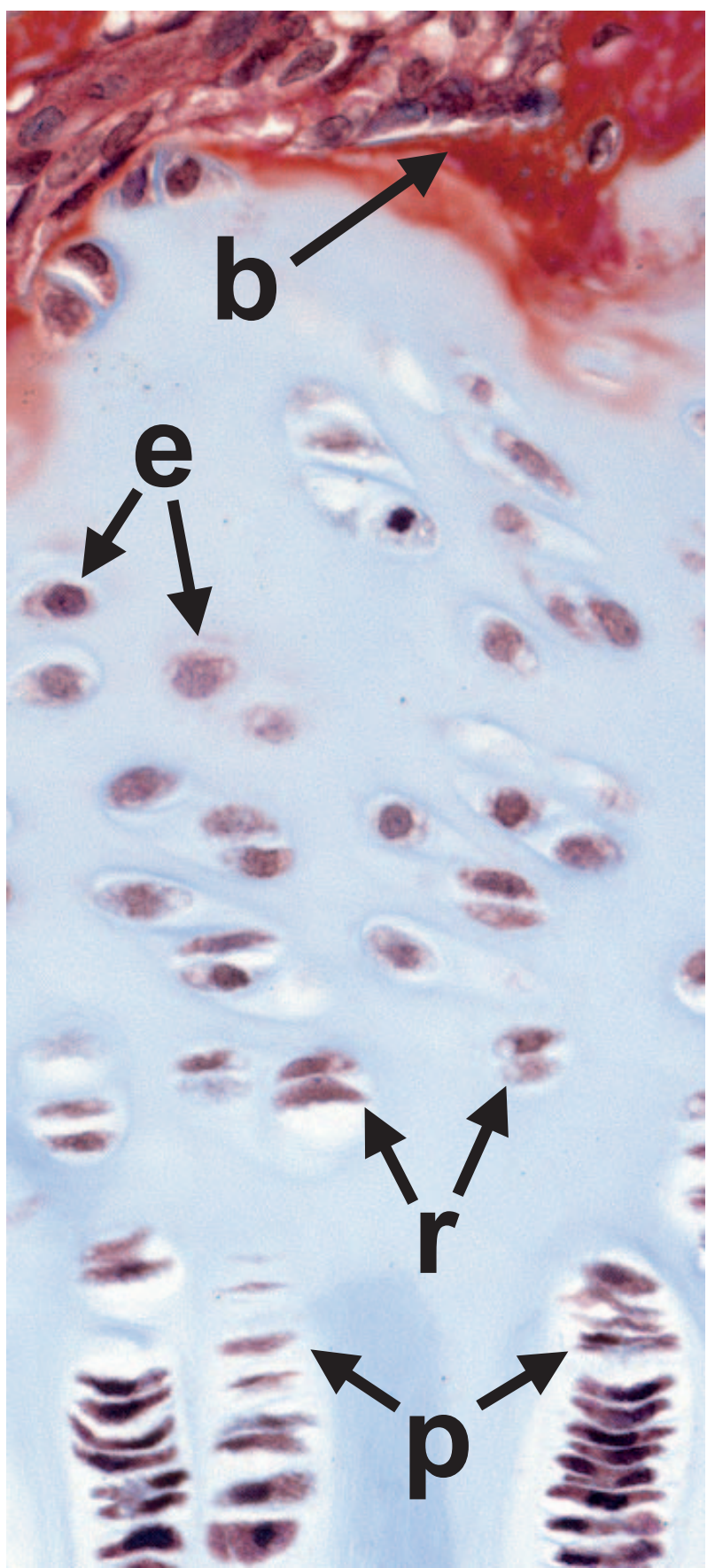

Figure 1 Location of reserve and epiphyseal chondrocytes in the resting zone. Reserve chondrocytes ( $r$ ) are flattened and often grouped in pairs aligned parallel to the long axis of the bone. Epiphyseal chondrocytes (e) are round or elliptical and mostly scattered individually in the cartilage matrix. Representative longitudinal section of resting zone of 6 -week-old male NZW rabbit stained with Masson Trichome is shown. (p), proliferative chondrocyte; (b), bone tissue. 


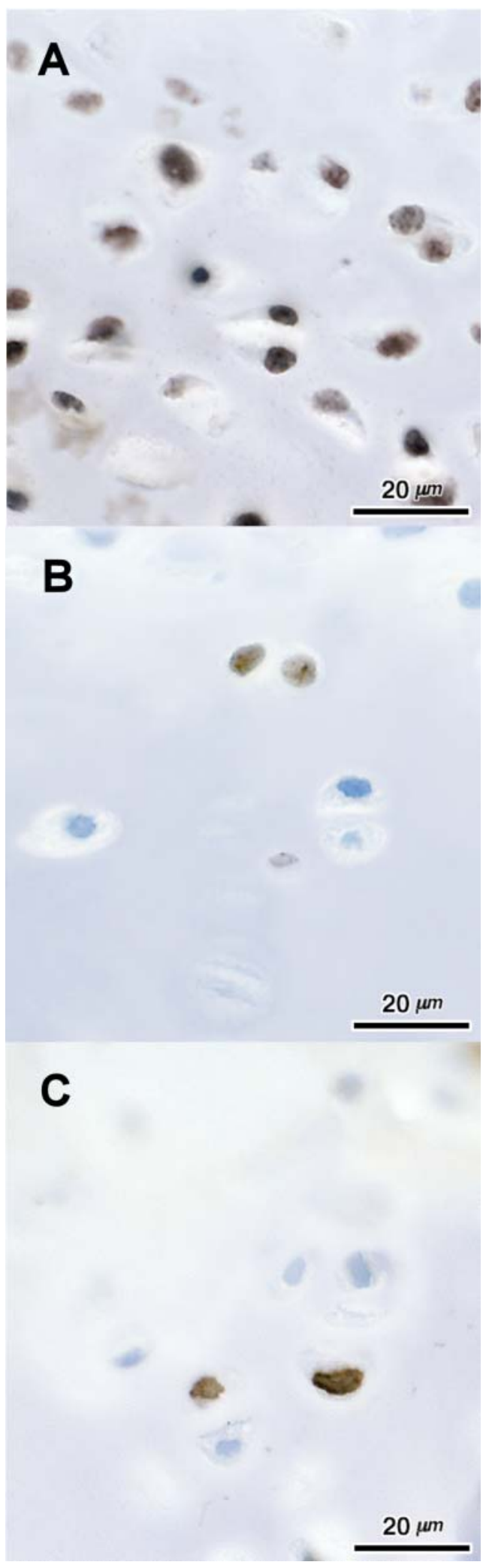

www.endocrinology-journals.org
To study the effect of age on resting zone proliferation in vivo, animals were studied at the end of gestation, and at postnatal week 5,9 , and 17 . The first time point represents the initial period of very rapid longitudinal bone growth. For simplicity, we refer to this time point as 0 weeks of age. The remaining time points represent the subsequent period of progressively decreasing growth (Masoud et al. 1986b, Nilsson et al. 2005). To avoid the confounding effects of gonadal steroids, rabbits studied at 9 and 17 weeks of age were previously castrated at 6 weeks of age. Five-week-old animals remained intact because they are sexually immature. Because resting zone cells replicate slowly, we made the measurement of proliferation rate by prolonged, continuous labeling of newly synthesized DNA using 5-bromo-2'-deoxy-uridine (BrdU). Pregnant females (gestational age 22 days, $n=2$ ) and 5-, 9-, and 17-week-old New Zealand White male rabbits $(n=6-8$ per group) received a subcutaneous osmotic minipump (2 ML-1; Alza Corp, Palo Alto, CA, USA) delivering BrdU (Sigma-Aldrich Corp.) suspended in physiological saline at a rate of $200 \mu \mathrm{g} B r d U / \mathrm{kg} / \mathrm{h}$. After the one-week infusion period, animals were killed by pentobarbital overdose. The pregnant females were killed at 29 days gestation, and a total of twelve fetuses were studied. Growth plate collections were made from the distal femur (DF), proximal tibia (PT), and distal tibia (DT). Growth plates were fixed in 10\% phosphate-buffered formalin for $24 \mathrm{~h}$, decalcified in $0.5 \mathrm{M}$ EDTA, placed in $70 \%$ ethanol for storage, and embedded in paraffin, after which longitudinal $5 \mu \mathrm{m}$ sections were obtained from the center of the bone.

To study the effect of dexamethasone and estrogen on resting zone proliferation, animals received either estradiol cypionate (Pharmacia \& Upjohn Company, Kalamazoo, MI, USA), $70 \mu \mathrm{g} / \mathrm{kg}$ i.m. weekly, dexamethasone (Phoenix Pharmaceutical, St Joseph, MO, USA), $0.5 \mathrm{mg} / \mathrm{kg}$ s.c. daily or vehicle (cottonseed oil im weekly as vehicle for estradiol cypionate and normal saline s.c. daily as vehicle for dexamethasone) for two weeks ( $n=6-7$ per treatment group). This dose of estradiol cypionate produces nearphysiological adult levels (Weise et al. 2001). This dose of dexamethasone is supraphysiological and has previously been shown to inhibit growth (Gafni et al. 2001) and cause osteoporosis (Gafni et al. 2002). Animals were weaned at 3 weeks of age. Treatment was begun at 4 weeks of age and lasted 2 weeks. After the first week of treatment, all animals underwent minipump implantation to deliver $\mathrm{BrdU}(200 \mu \mathrm{g} \mathrm{BrdU} / \mathrm{kg}$ per hour). After a total of 2 weeks treatment, animals were killed by pentobarbital overdose.

Figure 2 Proliferation in resting zone chondrocytes assessed by BrdU-labeling (brown color). Fetal (A), 5-week-old (B), and 17-week-old (C) NZW rabbits received a 1-week infusion of BrdU using a subcutaneous osmotic pump. BrdU-labeled cells were detected by immunohistochemistry in longitudinal sections of the growth plates. Representative photomicrographs are shown. 
Blood was drawn prior to sacrifice for measurement of estradiol and insulin-like growth factor I (IGF-I). Growth plates were isolated as described previously.

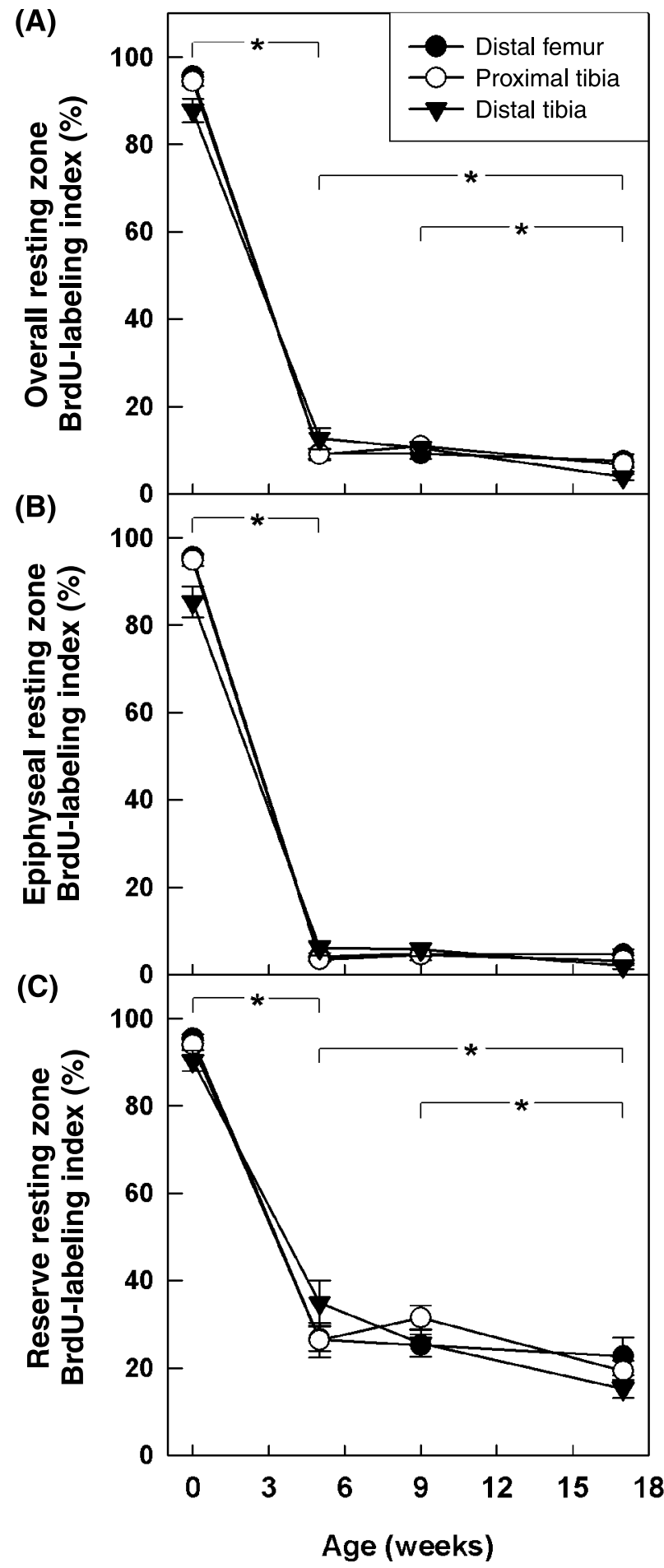

Immunohistochemistry

BrdU-labeled cells in growth plates were visualized by immunohistochemical staining using BrdU in situ detection kit II (BD Biosciences Pharmingen, San Diego, CA, USA). BrdU staining was performed as per the manufacturer's protocol, except that the antigen-retrieval time was extended to $15 \mathrm{~min}$. Subsequently, the antigen-retrieval solution was allowed to cool down to room temperature for $25 \mathrm{~min}$, and the incubation with anti-BrdU antibody was done overnight at $4{ }^{\circ} \mathrm{C}$. Sections were counterstained lightly with hematoxylin. As a negative control, sections of bone from animals not infused with BrdU were subjected to the same immunohistochemical procedure; no staining was observed.

\section{Cell kinetics}

In fetal animals, the resting zone can be subdivided into two regions, the reserve cartilage and the epiphyseal cartilage. The reserve cartilage lies closest to the proliferative zone and contains flattened chondrocytes. These chondrocytes are often grouped in pairs aligned parallel to the long axis of the bone. The epiphyseal cartilage lies further from the proliferative zone and thus closer to the bony epiphysis than the reserve cartilage. The epiphyseal cartilage contains round or elliptical chondrocytes, mostly scattered individually in the cartilage matrix (Abad et al. 2002). In older animals, both reserve chondrocytes and epiphyseal chondrocytes are far less numerous and they are more intermixed, without a distinct boundary (Fig. 1). We measured the BrdU-labeling index and numbers of cells in both subpopulations. Because the distinction is subjective, we also analyzed these measurements for the overall resting zone, including both cell types.

A single, blinded observer assessed cell proliferation by light microscopy. The central two-thirds of the growth plate was used for analysis; the lateral portions of the growth plate adjacent to the perichondrium were excluded. The BrdU-labeling index was defined as the number of BrdU-labeled cells divided by the total number of labeled and unlabeled cells (with visible nuclei, counted using phase contrast). The calculation of the labeling index in each growth plate was based on at least 300 cells. We also assessed the number of resting zone cells per millimeter growth plate, measured perpendicular to the

Figure 3 Effect of age on the proliferation rate of chondrocytes in the overall resting zone (A), and in the epiphyseal region (B), and the reserve region $(\mathrm{C})$ of the resting zone. 0 - (late fetal), 5-, 9-, and 17-week-old rabbits ( $n=6-12$ per group) were infused with BrdU for one week. BrdU labeling was assessed immunohistochemically in distal femur - proximal tibia $\bigcirc$, and distal tibia $\boldsymbol{\nabla}$. The BrdU-labeling index represents the number of BrdU-labeled cells divided by the total number of labeled and unlabeled cells. There is an overall effect of age for all three panels. $P<0 \cdot 001$, ANOVA; ${ }^{*} P \leq 0 \cdot 001$ for pairwise comparison. 
long axis of the bone. This value reflects, in three dimensions, the number of resting zone chondrocytes in the growth plate, divided by the circular area of the growth plate.

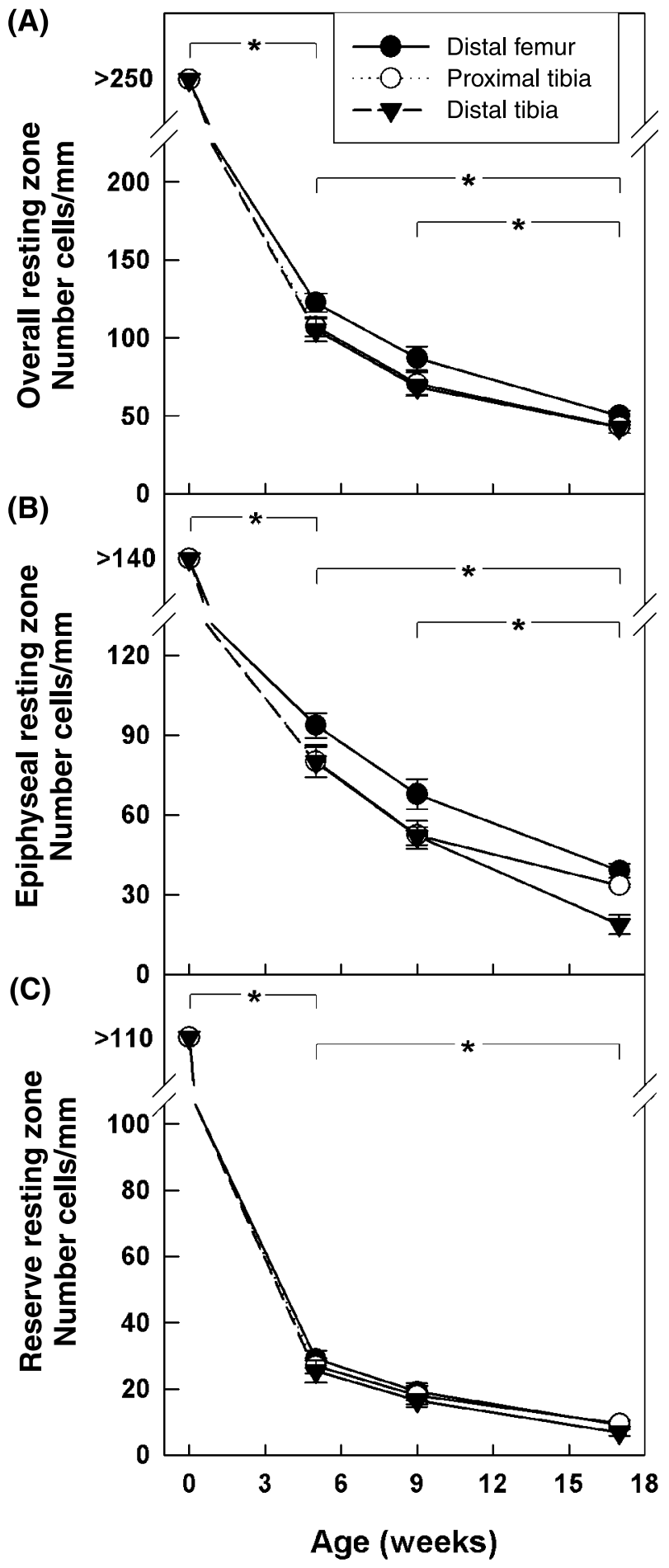

Assays

Serum IGF-I and estradiol concentrations were measured by radioimmunoassay at Esoterix Inc. (Austin, Texas, USA).

\section{Statistical analysis}

Data are presented as mean \pm S.E.M. The effects of age, estrogen, and dexamethasone on resting zone proliferation and on number of cells were evaluated by two-way repeated-measures ANOVA. For the effect of age, the ANOVA was followed by Holm-Sidak tests for pairwise multiple comparison. We chose not to analyze differences among different growth plates because these comparisons would have had less statistical power than the overall comparisons due to smaller sample size and the need to correct for multiple comparisons.

\section{Results}

\section{Effects of age}

The BrdU-labeling index in the resting zone decreased significantly with age $(P<0 \cdot 001$, Fig. 2 and 3$)$. For example, in the distal femur the labeling index decreased from $95 \cdot 6 \pm 0 \cdot 8 \%$ at 0 weeks of age to $9 \cdot 2 \pm 1 \cdot 2 \%$ at 5 weeks, $9 \cdot 2 \pm 1 \cdot 1 \%$ at 9 weeks, and $7 \cdot 6 \pm 1 \cdot 5 \%$ at 17 weeks of age. We also subdivided the resting zone into the epiphyseal region and the reserve resting zone. The BrdU-labeling index decreased with age in both regions $(P<0 \cdot 001$, Fig. 3B and C), although, in the epiphyseal region, a significant decrease was only observed during the $0-5$ week interval.

The 1-week continuous infusion of BrdU labeled essentially all proliferative chondrocytes, thus confirming that the proliferation rate in proliferative zone chondrocytes is more rapid than in resting zone chondrocytes. The hypertrophic chondrocytes were also all labeled, presumably because proliferative chondrocytes were labeled and then underwent terminal differentiation to become hypertrophic chondrocytes. We could confirm that this widespread labeling in the proliferative zone and the hypertrophic zone was not artifactual because it was not seen in control animals that did not receive BrdU,

Figure 4 Effect of age on the number of chondrocytes in the overall resting zone (A), and in the epiphyseal region (B), and the reserve region $(\mathrm{C})$ of the resting zone. The number of cells per $\mathrm{mm}$ of growth plate (measured perpendicular to the long axis of the bone) was assessed in the distal femur $\bullet$, proximal tibia $\bigcirc$, and distal tibia $\boldsymbol{\nabla}$ of 0- (late fetal), 5-, 9-, and 17-week-old rabbits ( $n=6-12$ per group). In the fetal rabbit, the number of cells is shown only as a lower limit because, at this age, there is no secondary ossification center to form an upper margin of the resting zone and therefore the resting zone extended beyond the counting area. There is an overall effect of age for all three panels. $P<0 \cdot 001$, ANOVA; * $P<0.001$ for pairwise comparison. 
and because a single injection of $\operatorname{BrdU}$ produced more selective labeling of proliferative zone chondrocytes and no labeling of hypertrophic zone chondrocytes (data not shown).

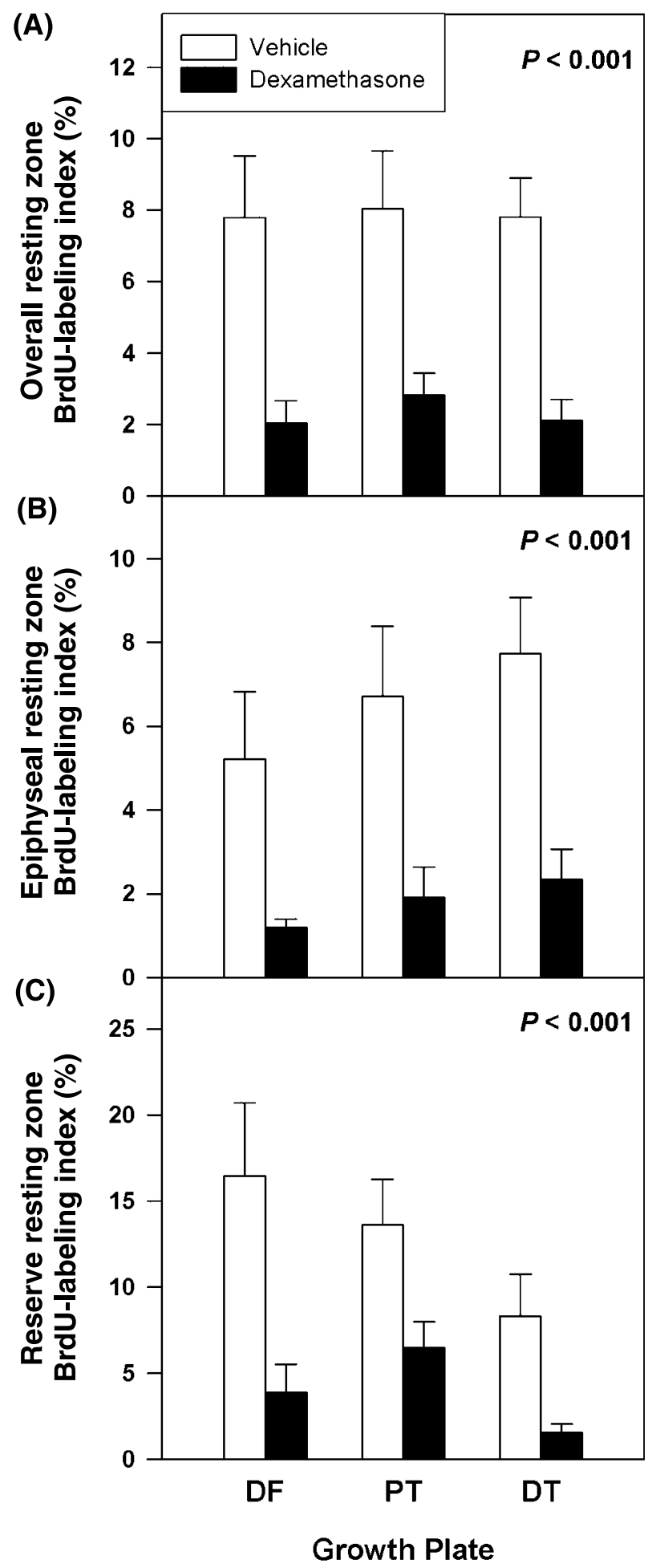

The number of resting zone chondrocytes per $\mathrm{mm}$ of growth plate also decreased with age in the overall resting zone $(P<0 \cdot 001$, Fig. 4A), in the epiphyseal resting zone $(P<0 \cdot 001$, Fig. 4B), and in the reserve resting zone $(P<0 \cdot 001$, Fig. 4C).

\section{Effects of dexamethasone and estradiol}

To gain insight into the effect of hormones on the resting zone, 4-week-old rabbits were treated with dexamethasone, estradiol, or vehicle for two weeks. BrdU-labeling was performed during the second week of the treatment.

The BrdU-labeling index in the resting zone decreased with dexamethasone treatment $(P<0 \cdot 001$, Fig. 5A). This decrease was seen in both the epiphyseal $(P<0 \cdot 001$, Fig. 5B) and the reserve resting zone $(P<0 \cdot 001$, Fig. $5 \mathrm{C})$. The number of resting zone chondrocytes was greater with dexamethasone treatment $(P=0 \cdot 016$, Fig. 6A). This effect was detected in the reserve resting zone $(P<0 \cdot 001$, Fig. 6C), but not in the epiphyseal resting zone (Fig. 6B). Dexamethasone treatment did not significantly affect serum IGF-I concentration $(112 \pm 8 \mathrm{ng} / \mathrm{mL}$ vs $108 \pm$ $6 \mathrm{ng} / \mathrm{mL}$, dexamethasone vs control, $P=\mathrm{NS}$ ).

The BrdU-labeling index in the resting zone was decreased by estrogen treatment $(P=0 \cdot 011$, Fig. 7A). This decrease occurred in the epiphyseal resting zone $(P=0 \cdot 008$, Fig. 7B), but the decrease did not reach statistical significance in the reserve resting zone $(P=0 \cdot 06$, Fig. 7C). Estrogen treatment did not significantly affect the number of resting zone chondrocytes (data not shown). Serum estradiol concentration, measured 7 days after the second injection of estradiol cypionate, was $11 \pm$ $2 \mathrm{pg} / \mathrm{mL}$, compared to $<5 \mathrm{pg} / \mathrm{mL}$ in animals treated with the vehicle. Estrogen treatment did not significantly affect serum IGF-I concentration $(88 \pm 6 \mathrm{ng} / \mathrm{mL}$ vs $108 \pm$ $6 \mathrm{ng} / \mathrm{mL}$, estrogen vs control, $P=\mathrm{NS}$ ).

\section{Discussion}

We found that the proliferation rate of cells in the resting zone, both the epiphyseal and reserve regions, decreased with age. This finding supports our hypothesis that the proliferative capacity of the stem-like cells in the resting zone decreases with age, and thus the stem-like cells become depleted qualitatively. This decrease in

Figure 5 Effect of dexamethasone on proliferation of chondrocytes in the overall resting zone $(\mathrm{A})$, and in the epiphyseal region $(\mathrm{B})$, and the reserve region $(\mathrm{C})$ of the resting zone. 4-week-old rabbits were treated with dexamethasone $(0.5 \mathrm{mg} / \mathrm{kg} \mathrm{sc}$ daily $)$ or vehicle (normal saline) for two weeks. BrdU was infused during the second week of the treatment. BrdU-labeling was assessed immunohistochemically in the distal femur (DF), proximal tibia (PT), and distal tibia (DT). $P$ values are for dexamethasone vs vehicle, ANOVA. 
proliferative capacity could explain the decreased proliferation in the proliferative zone chondrocytes which are thought to be derived from the stem-like cells

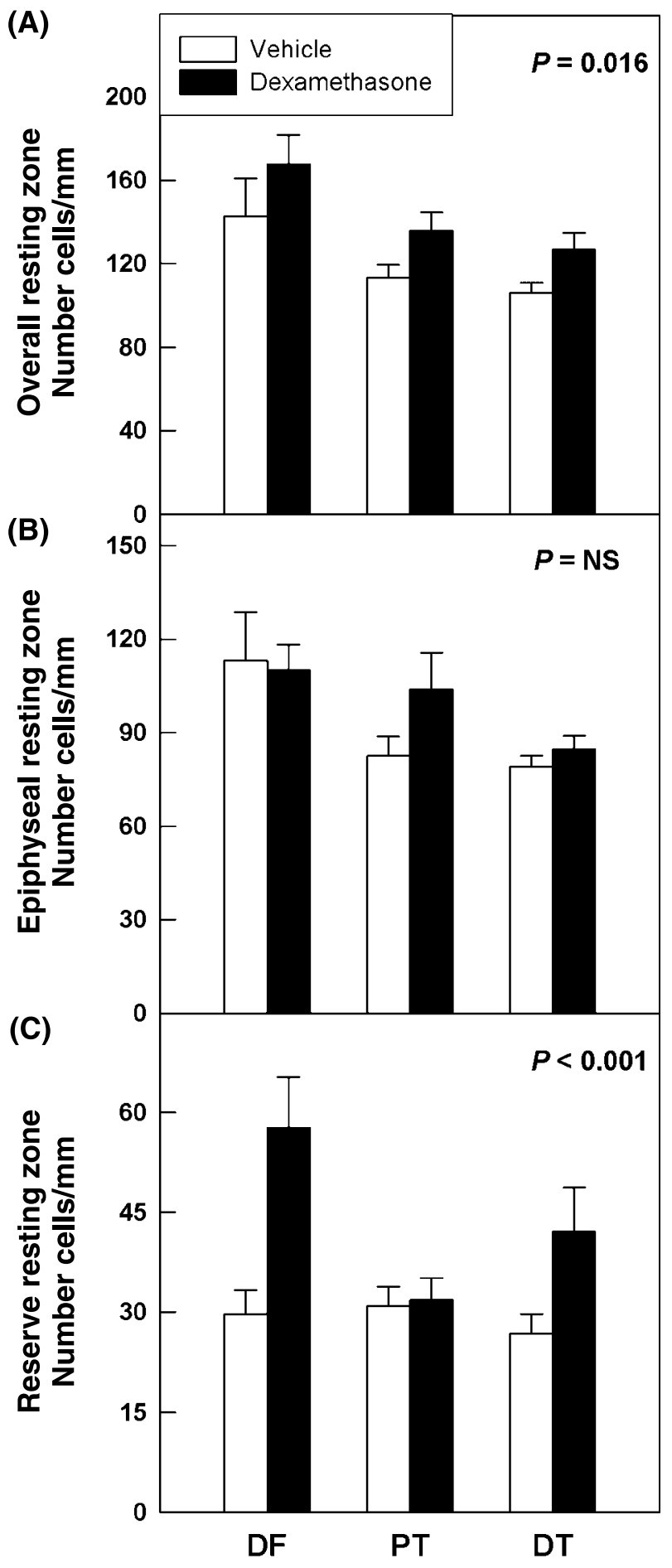

Growth Plate
(Abad et al. 2002), and thus could also explain the decline in growth rate with age.

Although we attribute the decrease in proliferation with age to a decrease in the proliferative capacity, this decreased capacity is not necessarily cell autonomous and irreversible. The decrease in proliferative capacity could be context-dependent, and reversible. Previous studies suggest that the decrease is not due to hormonal or other systemic factors (Nilsson \& Baron 2004), but the decrease could reflect a local change in concentrations of paracrine growth factors.

We also found that the number of resting zone chondrocytes decreased with age in both epiphyseal and reserve resting zone in the rabbit. Thus quantitative depletion of resting zone chondrocytes also occurred with age. This quantitative depletion could explain the reduction in column density that occurs with growth plate senescence (Weise et al. 2001), because with age there would be fewer stem-like cells to generate new columns, potentially increasing the spacing of the columns in the proliferative zone. If, with age, the resting zone generates proliferative columnar clones less often, the proliferative zone might be populated with older clones, which might also contribute to the decline in growth rate. Eventually, with age, the resting zone becomes essentially depleted of cells, after which time longitudinal bone growth can presumably only continue until the proliferative capacity of the existing clones of proliferative chondrocytes is exhausted. The decline in number of resting zone chondrocytes could also affect growth rate by a paracrine mechanism. For example, a decrease in the number of resting zone chondrocytes might decrease the overall production of parathyroid hormone-related protein (PTHrP), which could lead to earlier hypertrophy of proliferative zone chondrocytes.

The observed decrease in the number of resting chondrocytes with age presumably results, at least in part, from the observed decrease in proliferation of these cells. However, it is not known whether other factors may also contribute to this decrease in cell number, for example: an increase in the rate of cell death with age, an increase in the number of resting zone chondrocytes exiting to the proliferative zone, or an increase in the number of resting zone chondrocytes that hypertrophy and are then replaced in the secondary ossification center.

Previous studies have shown evidence that glucocorticoid excess slows the rate of growth plate senescence.

Figure 6 Effect of dexamethasone treatment on the number of chondrocytes per millimeter in the overall resting zone $(A)$, and in the epiphyseal region (B), and the reserve region (C) of the resting zone. 4-week-old rabbits were treated with dexamethasone $(0.5 \mathrm{mg} / \mathrm{kg} \mathrm{sc}$ daily) or vehicle (normal saline) for two weeks. The number of cells per $\mathrm{mm}$ of growth plate (measured perpendicular to the long axis of the bone) was assessed in the distal femur (DF), proximal tibia (PT), and distal tibia (DT). $P$ values are for dexamethasone vs vehicle, ANOVA. 


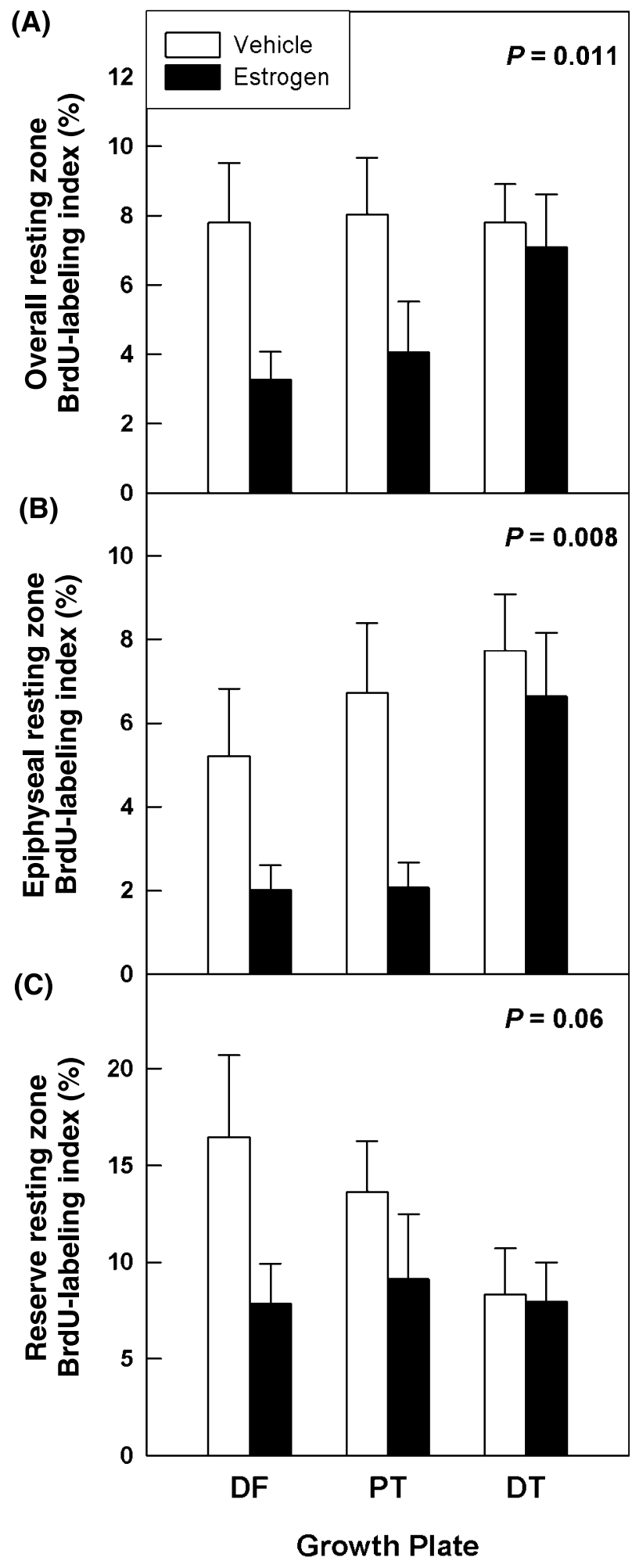

Journal of Endocrinology (2006) 189, 27-36
Consequently, after a period of glucocorticoid-induced growth inhibition, the growth plates appear less senescent than expected for age and therefore grow at a greater than normal rate, causing catch-up growth (Gafni et al. 2001). To explain the delay in senescence and the ensuing catch-up growth, we hypothesized that glucocorticoid excess decreases the proliferation rate in the resting zone, thus conserving the proliferative capacity of the resting zone chondrocytes. The data in the current study are consistent with this hypothesis; dexamethasone decreased proliferation in both the epiphyseal and the reserve resting zone.

In addition, dexamethasone-treated animals had more resting zone chondrocytes than did control animals, indicating that glucocorticoid slows the quantitative depletion of resting zone cells. This delayed depletion of stem-like cell in the resting zone could contribute to the slowing of senescence caused by glucocorticoid excess. This increased number of resting zone cells occurred despite decreased proliferation, suggesting that dexamethasone may slow the transition from resting to proliferative chondrocytes, thus conserving the pool of stem-like cells. We did not determine whether cessation of glucocorticoid treatment causes this transition from resting to proliferative chondrocytes to accelerate, which could contribute to catch-up growth.

Estrogen accelerates growth plate senescence (Weise et al. 2001). To explain this effect, we hypothesized that estrogen might accelerate resting zone chondrocyte proliferation, thus depleting the proliferative capacity more rapidly, or that estrogen might accelerate the quantitative depletion of chondrocytes from the resting zone. However, estrogen did neither; it decreased proliferation rate and had no significant effect on cell numbers. Thus, the mechanism by which estrogen accelerates senescence remains unclear.

We measured levels of circulating IGF-1. Although the decrease in serum IGF-1 due to estrogen was not statistically significant, we cannot exclude the possibility that a decrease in IGF-I might have contributed to the decrease in proliferation index. Also, we examined the effect of estrogen on the growth plate in male rabbits. The effect of estrogen might have been different in females; a sexually dimorphic response to estrogen has been demonstrated in rat growth plate chondrocytes in vitro (Ekstein et al. 2005).

Figure 7 Effect of estrogen on proliferation of chondrocytes in the overall resting zone $(A)$, and in the epiphyseal region $(B)$, and the reserve region $(\mathrm{C})$ of the resting zone. 4-week-old rabbits were treated with estradiol cypionate $(70 \mu \mathrm{g} / \mathrm{kg}$ im weekly) or vehicle (cottonseed oil) for two weeks. BrdU was infused during the second week of the treatment. BrdU-labeling was assessed immunohistochemically in the distal femur (DF), proximal tibia (PT), and distal tibia (DT). $P$ values are for estrogen vs vehicle, ANOVA. 
We speculate that estrogen might increase the loss of proliferative capacity that occurs with each cell cycle, or that estrogen might cause loss of proliferative capacity by a cell-cycle-independent mechanism. For example, if senescence is caused by epigenetic changes such as loss of DNA methylation with each cell cycle (Nilsson et al. 2005), then estrogen might act by decreasing expression of maintenance methylases, causing greater loss of methylation with each cell replication. The effects of estrogen on the resting zone chondrocytes could be mediated by the estrogen receptor- $\alpha$ or $-\beta$, both of which are expressed by resting zone chondrocytes in humans, rabbits, and rats (Nilsson et al. 2002, Nilsson et al. 2003). The combination of a decreased rate of growth (in most mammals) and an increased rate of senescence seems to be an effect specific to estrogen (De Luca \& Baron 1999). Other growthinhibiting conditions, such as glucocorticoid excess (Gafni et al. 2001) and hypothyroidism (Marino et al. 2005), decelerate growth plate senescence. The mechanism responsible for this paradoxical effect of estrogen remains to be determined.

In our study, we focused on the effects of estrogen on the resting zone and on growth plate senescence. However, estrogen may also act elsewhere in the growth plate to hasten epiphyseal fusion by other mechanisms. For example, estrogen may act on hypertrophic chondrocytes to stimulate fusion (Kennedy et al. 1999, Nilsson et al. 2002). In addition, signals other than estrogen may contribute to fusion, including fibroblast growth factors, (Sobue et al. 2005, Ornitz 2001), parathyroid hormonerelated protein (Schipani et al. 1997), and insulin receptor substrate-1 (Hoshi et al. 2004).

Although it was not one of the original aims of the study, we also observed that the proliferation rate of reserve resting zone chondrocytes was greater than the proliferation rate of epiphyseal resting zone chondrocytes at all ages studied except for 0 weeks of age. This finding supports the idea that the reserve chondrocytes represent a transition zone between the epiphyseal chondrocytes, which proliferate very slowly, and the proliferative chondrocytes, which proliferate rapidly. This concept is intuitively appealing in that the reserve region lies physically between the epiphyseal region and the proliferative zone. In addition, the reserve region contains pairs of flattened cells that give the appearance of nascent proliferative columns. Finally, the concept is supported by functional studies; after surgical ablation, the reserve region can apparently be regenerated by epiphyseal chondrocytes, and the proliferative zone can apparently be generated by reserve chondrocytes (Abad et al. 2002).

In conclusion, our findings support the hypotheses that growth plate senescence is caused by qualitative and quantitative depletion of stem-like cells in the resting zone. Our findings further suggest that growth-inhibiting conditions, such as glucocorticoid excess, slow senescence by slowing resting zone proliferation and slowing the numerical depletion of stem-like cells, thereby conserving the proliferative capacity of the growth plate. Estrogen appears to accelerate growth plate senescence without accelerating resting zone chondrocyte proliferation or accelerating the numerical depletion of these cells, suggesting that it might accelerate senescence by a proliferation-independent mechanism or by increasing the loss of proliferative capacity per cell cycle.

\section{Acknowledgements}

We thank Dr. John Bacher for performing the required surgical procedures. This research was supported by the Intramural Research Program of the National Institute of Child Health and Human Development, NIH. The authors declare that there is no conflict of interest that would prejudice the impartiality of this scientific work.

\section{References}

Abad V, Meyers JL, Weise M, Gafni RI, Barnes KM, Nilsson O, Bacher JD \& Baron J 2002 The role of the resting zone in growth plate chondrogenesis. Endocrinology 143 1851-1857.

De Luca F \& Baron J 1999 Control of bone growth by fibroblast growth factors. Trends in Endocrinology and Metabolism 10 61-65.

Ekstein J, Nasatzky E, Boyan BD, Ornoy A \& Schwartz Z 2005 Growth-plate chondrocytes respond to $17 \beta$-estradiol with sex-specific increases in IP3 and intracellular calcium ion signalling via a capacitative entry mechanism. Steroids 70 775-786.

Farnum CE \& Wilsman NJ 1987 Morphologic stages of the terminal hypertrophic chondrocyte of growth plate cartilage. Anatomical Record 219 221-232.

Farquharson C \& Loveridge N 1990 Cell proliferation within the growth plate of long bones assessed by bromodeoxyuridine uptake and its relationship to glucose 6-phosphate dehydrogenase activity. Bone and Mineral 10 121-130.

Gafni RI, Weise M, Robrecht DT, Meyers JL, Barnes KM, De-Levi S \& Baron J 2001 Catch-up growth is associated with delayed senescence of the growth plate in rabbits. Pediatric Research 50 618-623.

Gafni RI, McCarthy EF, Hatcher T, Meyers JL, Inoue N, Reddy C, Weise M, Barnes KM, Abad V \& Baron J 2002 Recovery from osteoporosis through skeletal growth: early bone mass acquisition has little effect on adult bone density. FASEB Journal $16736-738$

Hoshi K, Ogata N, Shimoaka T, Terauchi Y, Kadowaki T, Kenmotsu S-I, Chung UI, Ozawa H, Nakamura K \& Kawaguchi H 2004 Deficiency of insulin receptor substrate-1 impairs skeletal growth through early closure of epiphyseal cartilage. Journal of Bone and Mineral Research 19 214-223.

Hunziker EB \& Schenk RK 1989 Physiological mechanisms adopted by chondrocytes in regulating longitudinal bone growth in rats. Journal of Physiology 414 55-71.

Kember NF 1973 Aspects of the maturation process in growth cartilage in the rat tibia. Clinical Orthopedics and Related Research 95 288-294.

Kember NF \& Walker KV 1971 Control of bone growth in rats. Nature 229 428-429.

Kennedy J, Baris C, Hoyland JA, Selby PL, Freemont AJ \& Braidman IP 1999 Immunofluorescent localization of estrogen receptor- $\alpha$ in growth plates of rabbits, but not in rats, at sexual maturity. Bone 24 9-16. 
Marino R, Hegde AA, Nilsson O, Barnes KM, Emons JAM, Schrier L \& Baron J 2005 Catch-up growth after transient hypothyroidism is associated with delayed growth plate senescence. Proceedings of the $87^{\text {th }}$ Annual Meeting of the Endocrine Society, pp 220. San Diego, California, USA.

Masoud I, Shapiro F \& Moses A 1986a Tibial epiphyseal development: a cross-sectional histologic and histomorphometric study in the New Zealand white rabbit. Journal of Orthopedic Research 4 212-220.

Masoud I, Shapiro F, Kent R \& Moses A 19866 A longitudinal study of the growth of the New Zealand white rabbit: cumulative and biweekly incremental growth rates for body length, body weight, femoral length, and tibial length. Journal of Orthopedic Research 4 221-231.

National Research Council 2003 Guide for the Care and Use of Laboratory Animals. National Academy Press: Washington, DC, USA.

Nilsson O \& Baron J 2004 Fundamental limits on longitudinal bone growth: growth plate senescence and epiphyseal fusion. Trends in Endocrinology and Metabolism 15 370-374.

Nilsson O, Abad V, Chrysis D, Ritzen EM, Savendahl L \& Baron J 2002 Estrogen receptor-alpha and -beta are expressed throughout postnatal development in the rat and rabbit growth plate. Journal of Endocrinology 173 407-414.

Nilsson O, Chrysis D, Pajulo O, Boman A, Holst M, Rubinstein J, Ritzen EM \& Savendahl L 2003 Localization of estrogen receptors-alpha and -beta and androgen receptor in the human growth plate at different pubertal stages. Journal of Endocrinology 177 319-326.

Nilsson O, Mitchum Jr RD, Schrier L, Ferns SP, Barnes KM, Troendle JF \& Baron J 2005 Growth plate senescence is associated with loss of DNA methylation. Journal of Endocrinology 186 241-249.
Ornitz DM 2001 Regulation of chondrocyte growth and differentiation by fibroblast growth factor receptor 3. Novartis Foundation Symposium 232 272-282.

Schipani E, Lanske B, Hunzelman J, Luz A, Kovacs CS, Lee K, Pirro A, Kronenberg HM \& Juppner H 1997 Targeted expression of constitutively active receptors for parathyroid hormone and parathyroid hormone-related peptide delays endochondral bone formation and rescues mice that lack parathyroid hormone-related peptide. PNAS 94 13689-13694.

Sobue T, Naganawa T, Xiao L, Okada Y, Tanaka Y, Ito M, Okimoto N, Nakamura T, Coffin JD \& Hurley MM 2005 Over-expression of fibroblast growth factor-2 causes defective bone mineralization and osteopenia in transgenic mice. Journal of Cellular Biochemistry 95 83-94.

Tanner JM \& Davies PS 1985 Clinical longitudinal standards for height and height velocity for North American children. Journal of Pediatrics 107 317-329.

Walker KV \& Kember NF 1972a Cell kinetics of growth cartilage in the rat tibia. I. Measurements in young male rats. Cell and Tissue Kinetics 5 401-408.

Walker KV \& Kember NF $1972 b$ Cell kinetics of growth cartilage in the rat tibia. II. Measurements during ageing. Cell and Tissue Kinetics 5 409-419.

Weise M, De-Levi S, Barnes KM, Gafni RI, Abad V \& Baron J 2001 Effects of estrogen on growth plate senescence and epiphyseal fusion. PNAS 98 6871-6876.

Received in final form 27 December 2005

Accepted 5 January 2006

Made available online as an Accepted Preprint

11 January 2006 